\title{
Keynote Symposium
}

\section{KS-1}

Saving Species Through Cryobiology - Filling the Gap Between Technology and Ecology. PIERRE COMIZZOLI. Smithsonian Conservation Biology Institute, National Zoological Park, Washington DC. Email: comizzolip@si.edu

The maintenance of sustainable populations in the wild and the preservation of the whole planet's biodiversity highly depend on the protection of natural habitats and the advanced knowledge of animal and plant biology. Conservation biology is a multidisciplinary science that includes the creation of organized and accessible collections of biomaterials (Genome Resource Banks, GRB). For several decades, research institutions and zoos have been collecting and storing frozen samples, including raw DNA, somatic cells, tissues, blood products, germplasm (sperm and eggs) and embryos as well as other animal, plant and even soil products. These large cryo-collections are invaluable tools for understanding, cataloging, and protecting the genetic diversity of the world's unique animals and plants. Frozen biorepositories significantly contribute to scientific research and production of new scholarly knowledge in areas such as biodiversity genomics, taxonomy, phylogenetics, physiology, cellular and molecular biology, toxicology, and pathology. Biobanks also are actively used for endangered species management and propagation (including for species reintroduction). Importantly, the science of cryobiology offers the possibility to preserve the integrity of valuable samples for extended periods of time. Similar to the highly diverse biological traits observed across species, there is a huge species-specificity in cryobiology, especially for living biomaterials such as germplasms, cells, and tissues. It therefore is important to study and compare the intricacies and specificities associated with the successful cryopreservation, storage, and thawing of different biomaterials among different species. This knowledge also is essential to the successful use of these valuable bioresources for assisted reproduction and new biotechnologies (genomics, transcriptomics, and proteomics) that provide enormous amount of information. Biobanking can be considered as a science because collecting and preserving any possible samples from any species is now necessary for successful conservation biology. For instance, it will be soon possible to use any cell source to derive germ cells and produce embryos. Also, biomaterials may contain information that we are not able to extract and interpret yet. Thus, large amounts of samples have to be (1) systematically collected and preserved in optimal conditions and then (2) easily accessible to scientists. In addition, GRB efforts have to be financially sustainable and ensure that all existing specimens are acquired, safeguarded, exchanged and used in accordance with the policies and current best scientific and curatorial practices (which involves biosecurity, database harmonization, and easy access). There are good examples of GRB efforts fully integrated to conservation efforts (preservation and restoration of black-footed ferret populations for instance). Unfortunately, cryobiology, cryo-banking, and the appending technologies to use and analyze the samples are sometimes considered having relatively few impacts on conservation actions. It therefore is fundamental to continue building more bridges with other conservation biology disciplines. The priorities are to (1) increase the profile and demonstrate the value of frozen biorepositories to the broad conservationist community as well as the public, (2) establish a mechanism for effective communication/collaboration among disparate stakeholders using or interested in frozen biomaterials, (3) train more students and professionals in cryobiology and biobanking, (4) improve the financial sustainability for collection maintenance and growth, and (5) explore new preservation strategies to collect, process, store, and even use biomaterials in field conditions and at a lower cost.

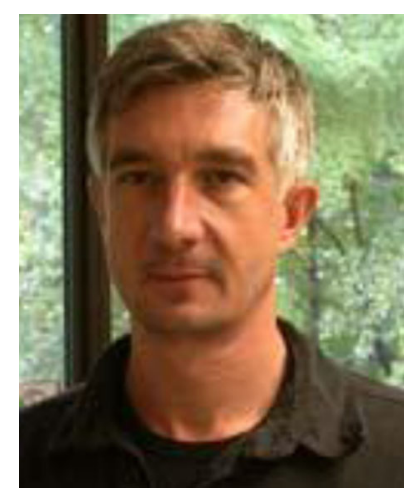

Pierre Comizzoli 